\title{
A hybrid substratum for primary hepatocyte culture that enhances hepatic functionality with low serum dependency
}

This article was published in the following Dove Press journal:

International Journal of Nanomedicine

23 March 2015

Number of times this article has been viewed

\author{
Qingyuan Meng ${ }^{1-3}$ \\ Chunsheng Tao ${ }^{1,4}$ \\ Zhiye Qiu' \\ Toshihiro Akaike ${ }^{3}$ \\ Fuzhai Cui' \\ Xiumei Wang'
}

'School of Materials Science and Engineering, Tsinghua University, Beijing, People's Republic of China; ${ }^{2}$ State Key Laboratory of Molecular Developmental Biology, Institute of Genetics and Developmental Biology, Chinese Academy of Sciences, Beijing, People's Republic of China; ${ }^{3}$ Biomaterials Center for Regenerative Medical Engineering, Ibaraki, Japan;

${ }^{4}$ The 40I Hospital of Chinese People's Liberation Army, Qingdao, People's Republic of China
Correspondence: Xiumei Wang School of Materials Science and Engineering, Tsinghua University, Beijing 100084, People's Republic of China Tel +861062782966

Email wxm@mail.tsinghua.edu.cn

\begin{abstract}
Cell culture systems have proven to be crucial for the in vitro maintenance of primary hepatocytes and the preservation of hepatic functional expression at a high level. A poly-(N-pvinylbenzyl-4-O- $\beta$-D-galactopyranosyl-D-gluconamide) matrix can recognize cells and promote liver function in a spheroid structure because of a specific galactose-asialoglycoprotein receptor interaction. Meanwhile, a fusion protein, E-cadherin-Fc, when incubated with various cells, has shown an enhancing effect on cellular viability and metabolism. Therefore, a hybrid substratum was developed for biomedical applications by using both of these materials to combine their advantages for primary hepatocyte cultures. The isolated cells showed a monolayer aggregate morphology on the coimmobilized surface and displayed higher functional expression than cells on traditional matrices. Furthermore, the hybrid system, in which the highest levels of cell adhesion and hepatocellular metabolism were achieved with the addition of $1 \%$ fetal bovine serum, showed a lower serum dependency than the collagen/gelatin-coated surface. Accordingly, this substrate may attenuate the negative effects of serum and further contribute to establishing a defined culture system for primary hepatocytes.
\end{abstract}

Keywords: mouse primary hepatocytes, E-cadherin-Fc, PVLA, serum dependency, hybrid system

\section{Introduction}

Hepatocytes, which compose the majority of the cellular mass in the liver, play many complex roles in the metabolism of living organisms; these functions include detoxification, metabolism, bile secretion, immune defense, and protein synthesis. ${ }^{1}$ The features of isolated hepatocytes from adult mammals during in vitro primary incubation may reflect the general properties of these cells in vivo. Accordingly, a valuable cellular model has been established for studies regarding the regulation of the functionality, senescence, and transformation of liver cells. Because of their easy access, the cells are widely utilized in clinical trials to investigate hepatic function, estimate xenobiotic metabolism, establish artificial organs in vitro, and further regenerate tissue in vivo..$^{2,3}$ However, hepatocytes tend to lose their reproductive activity and specific liver function steadily after isolation. Meanwhile, attempts to replace primary cells by using permanent cells or even cancer cell lines have also encountered drawbacks because the substitute cells are not fully consistent with primary cells with regard to metabolism and functionality. Therefore, the use of a primary culture of hepatocytes remains a significant challenge, and various techniques have been devised to promote cellular viability and functional expression; these methods include the development of extracellular matrix (ECM) substrata, soluble factor supplements, and coculture systems. ${ }^{4,5}$ 
Appropriate modifications of physical and chemical methods for culture matrices may lead to the retention of the cellular replication capacity and differentiated functions in hepatocytes. Cell-recognizable materials have become increasingly available for the construction of cell culture substrata, which can mimic the in vivo conditions to provide specific ECMs for specific cell lineages to induce functional differentiation. Among these materials, poly-(Np-vinylbenzyl-4-O- $\beta$-D-galactopyranosyl-D-gluconamide) (PVLA) possesses galactose termini on its side chains and can specifically recognize hepatocytes through an interaction between asialoglycoprotein receptors (ASGPRs) on the cellular membrane and the polymer. ${ }^{6,7}$ ASGPR is a membrane receptor on liver cells, and its primary physiological function is to internalize galactose from circulating blood. ${ }^{8}$ It is further involved in the removal of apoptotic cells and acts as a carrier of low-density lipoprotein and as an entry point for hepatotropic viruses or DNA. ${ }^{9}$ ASGPR, in association with PVLA, assists primary hepatocytes in forming a multilayered aggregate morphology on the matrix. The liver-specific functions of the incubated cells on the galactose-carrying polymer, including ammonia removal and albumin secretion, have been found to be enhanced over the long term. ${ }^{10,11}$ Moreover, a chimeric protein, E-cadherin-Fc (EFC), composed of the E-cadherin extracellular domain and the immunoglobulin $\mathrm{G}$ (IgG) Fc domain, is capable of enhancing the cellular viability of endodermal cells. ${ }^{12}$ The protein-coated surface could further preserve the hepatocellular functions and metabolism of hepatic cell lineages. ${ }^{12,13}$ This combination in the culture system can promote the hepatic induction of embryonic stem cells in the late stage of oriented differentiation. ${ }^{14}$

In addition, primary hepatocytes were incubated with serum in early experiments to promote cellular behaviors. ${ }^{15}$ However, it is possible that the presence of fetal bovine serum (FBS) in the culture medium may produce a nonimmunosuppressive condition and introduce xenobiotic substances into the system. The supplementation of the serum can increase the risk of disease transmission, including spongiform encephalitis, when the cells are expanded for clinical transplantation. Because of its instability in composition and quality, serum supplementation may further lead to a reduction in cellular metabolism. ${ }^{16}$ In one experiment, cells were maintained in a hormonally defined medium because of their response to hepatotropic growth factors, including epidermal growth factor, transforming growth factor- $\alpha$, and insulin, to stabilize the culture system and reduce the negative influence. Although the cost of the incubation system, especially for massive cell culture, was increased, the cellular behaviors in this system were preserved or even promoted. Therefore, it is beneficial if the PVLA matrix does not require serum for hepatocyte maintenance and induction. ${ }^{17,18}$

In this study, isolated hepatocytes were seeded on a hybrid substrate of PVLA and EFC to achieve monolayer aggregate morphology in vitro. Increased functional and metabolic expression were observed in primary hepatocytes at both the gene and protein levels, unlike in the cells on collagen or gelatin surfaces. The effects of serum concentration $(0 \%$, $1 \%$, and $10 \%$ ) on cellular attachment, maintenance, and hepatic marker expression were further analyzed, and a low serum dependency in the cell-recognizable system with dual components was revealed.

\section{Materials and methods Preparation of cell-recognizable matrices}

PVLA and EFC were prepared by previously described methods and filter sterilized after dissolution. ${ }^{19,20}$ The hybrid substratum was produced by the sequential addition of EFC $(2.5 \mu \mathrm{g} / \mathrm{mL})$ and PVLA $(50 \mu \mathrm{g} / \mathrm{mL})$ solutions for 2 hours each to a nontreated polystyrene (PS) dish at $37^{\circ} \mathrm{C}$. The control substrata were produced by adding PVLA $(100 \mu \mathrm{g} / \mathrm{mL})$ solution to a nontreated dish or adding gelatin $(1 \mathrm{mg} / \mathrm{mL})$ or collagen I $(100 \mu \mathrm{g} / \mathrm{mL})$ solutions to treated PS dishes at $37^{\circ} \mathrm{C}$ for 2 hours each. The surfaces were rinsed thrice with phosphate buffered saline (PBS) and prepared for the future incubation of primary hepatocytes.

\section{Enzyme-linked immunosorbent assay (ELISA) for EFC adsorption}

The sample solutions of EFC were prepared at different concentrations consisting of $1,2,4,6,8,10,15$ and $30 \mu \mathrm{g} / \mathrm{mL}$. Then, $50 \mu \mathrm{L}$ of EFC solution at each concentration was maintained in a nontreated 96 -well plate for 2 hours at $37^{\circ} \mathrm{C}$. The surface was subsequently washed thrice with PBS and blocked by using $100 \mu \mathrm{L}$ of Blocking One solution (Nacalai Tesque, Kyoto, Japan) for 30 minutes. After the liquid was completely removed, $50 \mu \mathrm{L}$ of horseradish peroxidase (HRP)-conjugated anti-mouse IgG antibody (Jackson ImmunoResearch Laboratories, West Grove, PA, USA) was added, and the plate was incubated for 1 hour at room temperature. O-phenylenediamine dissolved in $100 \mu \mathrm{L}$ of citric acid-phosphoric acid solution with $\mathrm{H}_{2} \mathrm{O}_{2}$ was then added to the wells for 10 minutes at room temperature. Approximately $20 \mu \mathrm{L}$ of $\mathrm{H}_{2} \mathrm{SO}_{4}$ solution $(1 \mathrm{M}$ ) was added to the mixed solution to produce a $\mathrm{pH}$ value of 1.0 . Finally, the optical density was measured at $492 \mathrm{~nm}$ by using a microplate reader. 


\section{Quartz crystal microbalance (QCM) test for coimmobilization of EFC and PVLA}

The sequential adsorption of EFC and PVLA was confirmed by using the AFFINIX Q4 apparatus (Initium Co. Ltd., Tokyo, Japan) with a temperature controlling system. First, the electrodes of the QCM cells were cleaned and coated with a thin film of PS by using a high-speed spinning method. After the QCM instrument was stabilized for 2 hours, $500 \mu \mathrm{L}$ of EFC solution $(2.5 \mu \mathrm{g} / \mathrm{mL})$ was added to the cell. The surface was washed with distilled water after the oscillation frequency was stabilized. Subsequently, $500 \mu \mathrm{L}$ of PVLA solution $(50 \mu \mathrm{g} / \mathrm{mL})$ was added to the cell, which was then rinsed. The change of mass per unit area was calculated from the change in frequency by using the Sauerbrey Equation. A Langmuir adsorption curve was produced via double reciprocal data fitting.

\section{Isolation and culture of mouse primary hepatocytes}

The primary hepatocytes were obtained from the liver of anaesthetized male mice (6-7 weeks old) (Japan SLC Inc, Hamamatsu, Japan) by using a two-step hepatic portal vein perfusion method. ${ }^{21,22}$ All procedures complied with the institutional ethical use protocol of the Animal Center in Tokyo Institute of Technology. After removal, the tissue was dissected in Hank's solution and filtered through a $100 \mathrm{~mm}$ cell strainer. Then, the living hepatocytes were isolated and purified by using density gradient centrifugation. Subsequently, the cells were suspended in Gibco ${ }^{\circledR}$ Dulbecco's Modified Eagle's Medium (Thermo Fisher Scientific, Waltham, MA, USA) with FBS (volume/volume [v/v] $0 \%, 1 \%$, or $10 \%$ ) and antibiotics $(50 \mu \mathrm{g} / \mathrm{mL}$ penicillin, $50 \mu \mathrm{g} / \mathrm{mL}$ streptomycin, and $100 \mu \mathrm{g} / \mathrm{mL}$ neomycin). After the cellular viability was confirmed by using the Trypan Blue exclusion method $(>95 \%)$, the viable cells were seeded on previously coated PVLA, gelatin, collagen, and coimmobilized surfaces. The primary hepatocytes were incubated at $37^{\circ} \mathrm{C}$ in an atmosphere of $5 \% \mathrm{CO}_{2}$ during culture. The medium was changed after 4 hours to remove the nonadherent and dead cells and was changed every day thereafter during culture.

\section{Measurement of cell viability by using a cell adhesion assay}

After washing with PBS, the hepatocytes incubated on various coated surfaces in a 96-well plate were fixed with $4 \%$ formaldehyde (Wako Pure Chemical Industries, Ltd., Osaka, Japan) for 15 minutes and later stained with $0.1 \%$ (weight/ volume [w/v]) Crystal Violet for 10 minutes. The absorbency at $570 \mathrm{~nm}$ was measured after adding $2 \%(\mathrm{v} / \mathrm{v})$ sodium dodecyl sulfate solution for 30 minutes. Evaluations of cell attachment of the cultured cells were performed at 4 hours and subsequently every 2 days thereafter (days 1, 3, 5, 7, and 9) to estimate cell maintenance. The percentage of cultured cells that remained after $3,5,7$, and 9 days relative to the original number of the cells on the first day was considered as the relative preservation of isolated hepatocytes in each culture system.

\section{Semiquantitative reverse transcription polymerase chain reaction}

On days 1 and 5, RNA from the incubated hepatocytes in the hybrid systems with different serum concentrations was extracted from the cell lysates by using the TRIzol reagent (Thermo Fisher Scientific) suspended in distilled water; later, the RNA was reverse transcribed into complementary DNA. Then, polymerase chain reaction (PCR) was performed, and the products were analyzed by using $2 \%$ agarose gel electrophoresis and scanned by using a Molecular Dynamics Typhoon 8600 Imager. The relative intensities of the target genes (the genes for albumin, hepatocyte nuclear factor-4 alpha [HNF-4 $\alpha$ ], and ASGPR) were calculated with ImageJ image analysis software (Version 1.48) and were compared to a housekeeping marker, glyceraldehyde 3-phosphate dehydrogenase. The level of expression of these genes in cells on gelatin surfaces with $10 \%$ FBS served as a control.

\section{Immunofluorescence analysis of hepatic markers}

After 1 day and again after 5 days, PBS-rinsed primary hepatocytes in various systems were fixed with $8 \%$ formaldehyde for 20 minutes and were made permeable with $0.2 \%$ Triton X-100 (Nacalai Tesque) for 5 minutes. After blocking with Blocking One solution for 1 hour, the cells were incubated with primary antibodies to albumin (goat anti-mouse albumin; Abcam, Cambridge, UK) or ASGPR (rabbit anti-human ASGPR1/2; Santa Cruz Biotechnology, Dallas, TX, USA) for 2 hours at room temperature. The cells were triple-rinsed with PBS and incubated with the corresponding secondary antibodies (Alexa 555conjugated donkey anti-goat IgG, Thermo Fisher Scientific; Alexa 488-conjugated goat anti-rabbit IgG, Thermo Fisher Scientific) for 2 hours; the cells were then counterstained with 4',6-diamidino-2-phenylindole (Sigma-Aldrich, St Louis, MO, USA) to label the cell nuclei. After rinses with PBS, protein staining in the cells was examined with a fluorescent inverted microscope (Olympus Corporation, Tokyo, Japan). 


\section{Western blot analysis}

After 1 day and again after 5 days, the cellular proteins were extracted from the primary hepatocytes cultured on coimmobilized matrices with different serum concentrations. The cells cultured on gelatin with 10\% FBS were used as the control group. Then, the proteins were separated by sodium dodecyl sulfate-polyacrylamide gel electrophoresis and transferred onto a polyvinylidene diffuoride membrane (EMD Millipore, Billerica, MA, USA). The membrane was incubated with primary antibodies to albumin (Abcam) and $\beta$-actin (Sigma), the latter of which was used as a housekeeping marker, for 2 hours. After the rinses, the membrane was further incubated with an HRP-conjugated secondary antibody (Jackson ImmunoResearch Laboratories) for another 2 hours at room temperature. The HRP activity was assayed with Immobilon Western detection reagents (EMD Millipore) and observed under a fluorescent inverted microscope.

\section{Glycogen storage (periodic acid - Schiff reaction)}

After 3 days of culture, the primary hepatocytes were fixed with $8 \%$ formaldehyde for 10 minutes. After being rinsed with periodic acid - Schiff (PBS), the cells were oxidized in $1 \%$ periodic acid (Wako Pure Chemical Industries, Ltd.) for 5 minutes and then rinsed again. After the addition of the Schiff reagent (Wako Pure Chemical Industries, Ltd.) for 10 minutes, the cells were washed and observed.

\section{Statistical analysis}

The results are presented as the mean \pm standard deviation and were compared by using a Student's $t$-test. A $P$-value $<0.05$ was considered statistically significant, and a $P$-value $<0.01$ was considered highly statistically significant.

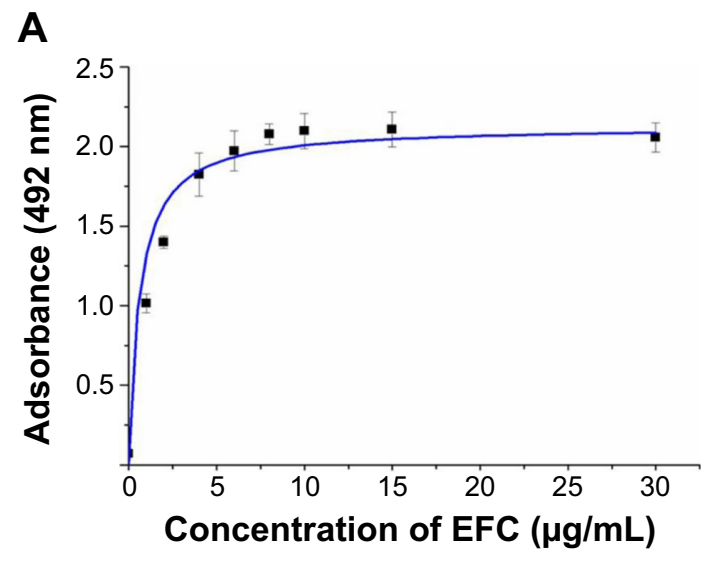

\section{Results}

The construction of a hybrid matrix

First, the EFC adsorption on the PS dish was confirmed by using the ELISA method, which indicated that the protein adsorption curve conformed to the Langmuir pattern. The lowest concentration of the protein solution to form a monolayer on PS surface was $10 \mu \mathrm{g} / \mathrm{mL}$ and was considered as the monolayer concentration (Figure 1A). Then, EFC and PVLA were successively added to a nontreated PS surface and evaluated by using the QCM method (Figure 1B). EFC with a concentration less than the monolayer concentration $(2.5 \mu \mathrm{g} / \mathrm{mL})$ was immobilized on a dish surface, and space was left for subsequent PVLA addition. Therefore, a hybrid matrix with two components was constructed for future applications in hepatocyte culture.

\section{The attachment of hepatocytes to the hybrid matrix}

The isolated hepatocytes maintained their spherical morphology on PVLA-coated dishes after 4 hours (Figure 2A), whereas their morphology was more spread out on control matrices consisting of gelatin and collagen (Figure 2C and D). Meanwhile, the primary hepatocytes showed a dual pattern. Some cells contracted while other cells were spread out because of the presence of both PVLA and EFC in the culture system (Figure 2B). Moreover, the serum requirement for the cells to achieve the maximal adherence on each surface differed in accordance with the substrata. In particular, on the hybrid matrix and on the PVLA surface, the number of hepatocytes was higher for the cells supplied with $1 \%$ FBS than for the cells supplied with serum at other concentrations (Figure 2E). The cells exhibited better attachment to

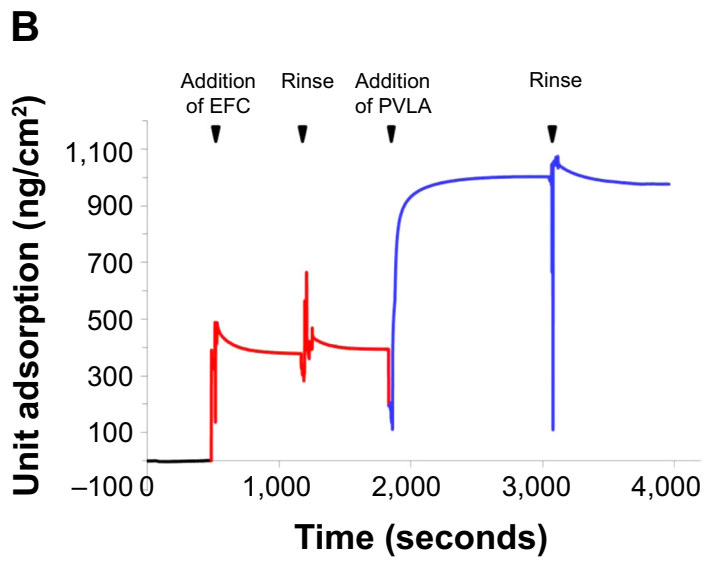

Figure I The adsorption of substrata on the PS surface.

Notes: The EFC adsorption of PS was confirmed by using the ELISA method, which revealed that $10 \mu \mathrm{g} / \mathrm{mL}$ was the monolayer concentration of EFC (A). The coimmobilization of EFC (red) and PVLA (blue) is shown (B).

Abbreviations: PS, polystyrene; EFC, E-cadherin-Fc; ELISA, enzyme-linked immunosorbent assay; PVLA, poly-(N-p-vinylbenzyl-4-O- $\beta$-D-galactopyranosyl-D-gluconamide). 

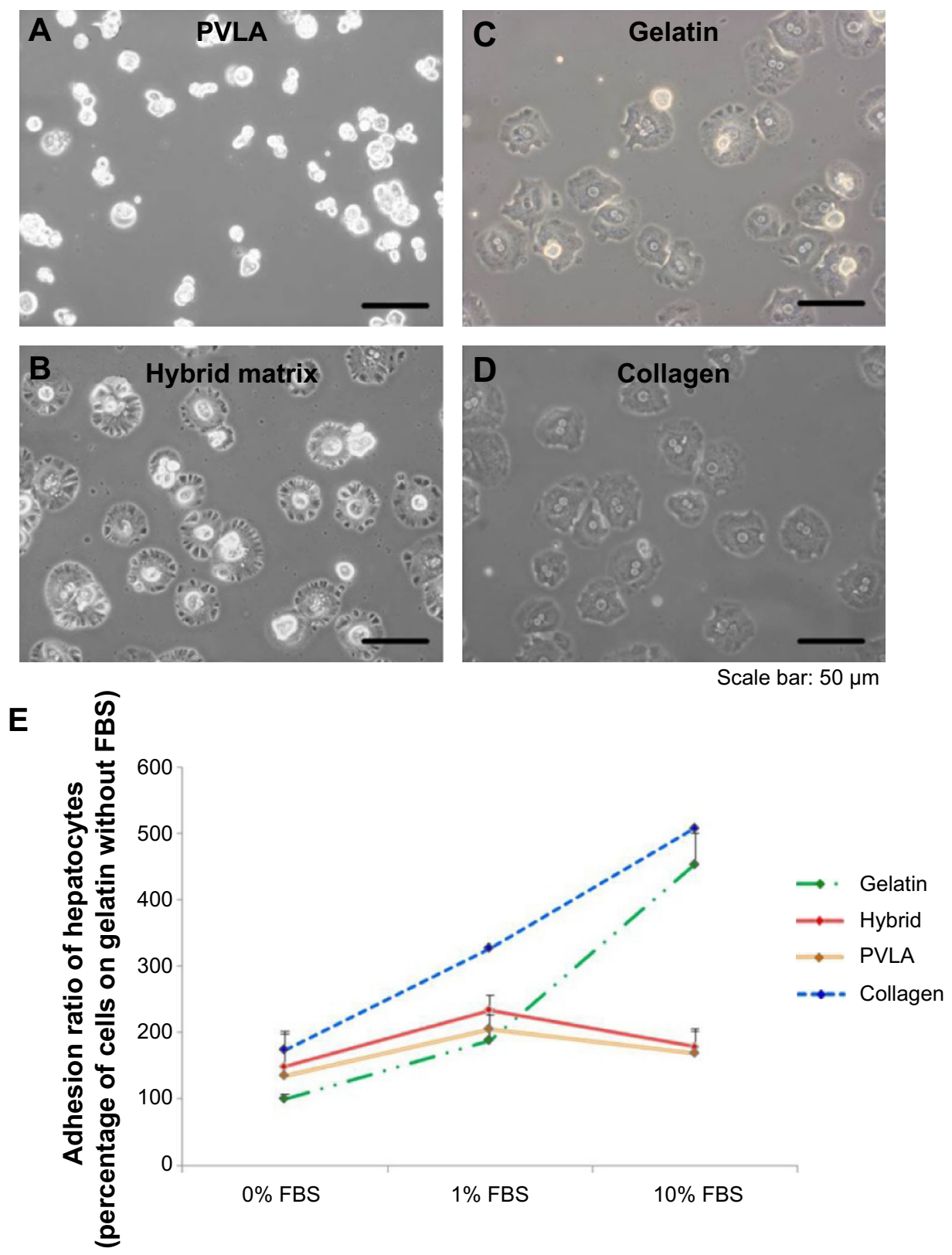

Figure 2 The attachment of primary hepatocytes in various systems (4 hours).

Notes: The cell morphology on PVLA with I\% FBS (A), the hybrid matrix with I\% FBS (B), gelatin with 10\% FBS (C), and collagen with 10\% FBS (D) is shown. The relative amount of attached hepatocytes was further determined by using the number of cells on the gelatin surface without $F B S$ as the $100 \%$ value (E). The cell numbers on gelatin and on collagen increased when the serum concentration ranged from $0 \%$ to $10 \%$, and they reached the highest level on the PVLA-containing matrices in the medium with I\% FBS. The hybrid matrix was made up of EFC and PVLA. Scale bar: $50 \mu \mathrm{m}$.

Abbreviations: FBS, fetal bovine serum; PVLA, poly-(N-p-vinylbenzyl-4-O- $\beta$-D-galactopyranosyl-D-gluconamide); EFC, E-cadherin-Fc.

traditional matrices, including collagen and gelatin, with a serum supply in the concentration range of approximately $0 \%-10 \%$ (Figure $2 \mathrm{E}$ ); further increases in serum concentration showed no additional beneficial influence. ${ }^{23}$ Aside from the hepatocytes on PVLA-coated dishes, the differences in cell viability on the other three matrices are statistically significant among the different FBS concentrations $(0 \%, 1 \%$, and $10 \%$ ) supplied for the cultures.

\section{The maintenance of hepatocytes on the hybrid matrix}

The matrices continued to show effects on cell behavior during hepatocyte maintenance in culture. One day after seeding, the primary hepatocytes revealed a monolayer morphology on coimmobilized (Figure 3A and G) and gelatincoated surfaces (Figure $3 \mathrm{C}$ and I), while they maintained a round shape on PVLA surfaces (Figure 3B and H). During 

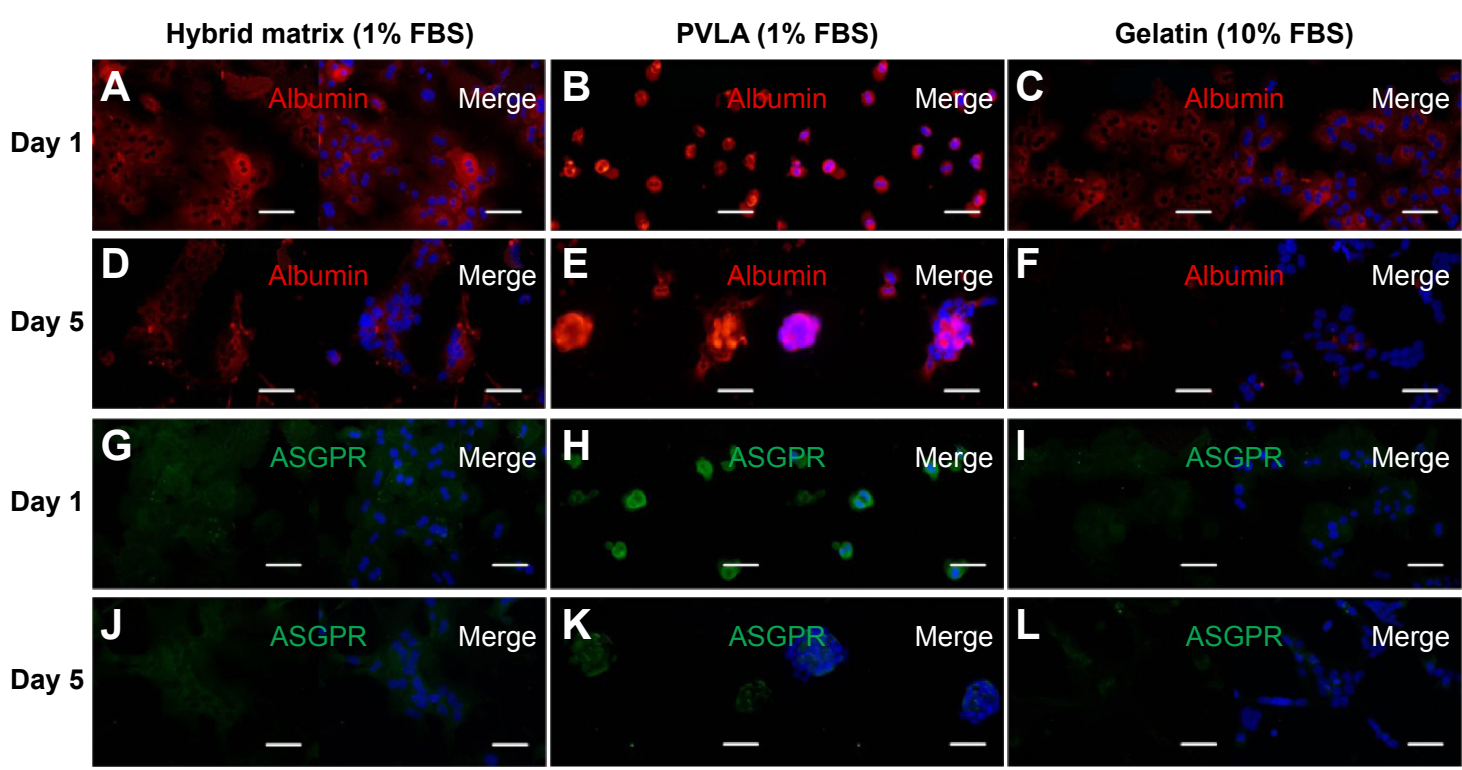

Scale bar: $50 \mu \mathrm{m}$

Figure 3 Immunofluorescence images of primary hepatocytes.

Notes: The immunofluorescence images of primary hepatocytes cultured on EFC-PVLA hybrid matrix, PVLA, and gelatin after I day and after 5 days. The expression of albumin (red, A-F) and ASGPR (green, G-L) is shown, and DAPI was used for nuclear staining (blue). Scale bar: $50 \mu \mathrm{m}$.

Abbreviations: FBS, fetal bovine serum; PVLA, poly-(N-p-vinylbenzyl-4-O- $\beta$-D-galactopyranosyl-D-gluconamide); ASGPR, asialoglycoprotein receptor; DAPI, 4',6-diamidino2-phenylindole; EFC, E-cadherin-Fc.

continuous incubation, the hepatocytes clustered in the culture systems because of the presence of PVLA, whereas the cells spread out on the gelatin and other traditional matrices. Finally, the cells formed monolayer aggregates on the hybrid matrix (Figure 3D and $\mathrm{J}$ ) and multilayer spheroid structures on PVLA-coated dishes (Figure 3E and K) after 5 days in culture. Furthermore, the viability of the maintained cells reached the highest level at each time point (days 1,3 , 5,7 , and 9) with a concentration of $1 \%$ FBS on the hybrid matrix and on PVLA (Figure 4A); however, a higher serum concentration could possibly enhance cell maintenance in the gelatin or collagen systems. The highest cell numbers, in ascending order for different time points, occurred on the following surfaces: PVLA $<$ hybrid surface $<$ gelatin and collagen, especially in the early culture period. The total number of cells gradually became smaller over time, and finally remained similar among these systems on days 7 and 9 . Moreover, the number of remaining hepatocytes relative to the initial cell number during 1 week was higher on the surfaces with PVLA than on the other matrices supplemented with the optimal serum concentrations (Figure 4B).

\section{The hepatic marker expression of hepatocytes on the hybrid matrix}

The specific markers expressed in primary hepatocytes were measured at both the protein and the gene levels by using various methods. Our results indicated that both the matrix and serum in the culture system may affect the cellular behavior of isolated hepatocytes. The immunofluorescence staining method revealed the expression of the proteins, including albumin and ASGPR, in primary hepatocytes. After 1 day, most of the isolated cells expressed albumin (red, Figure 3A-C) and ASGPR (green, Figure 3G-I), both in relatively high levels on various matrices, although different patterns were revealed that included both contracted and spread out morphology. The hepatocytes on the hybrid matrix and PVLA-coated dish further maintained albumin (red, Figure 3D and F) and ASGPR (green, Figure 3J and K) expression in large amounts after 5 days, whereas the amount of expression rapidly decreased on other matrices.

After 1 day and again after 5 days, the hepatic genes responsible for the liver function in cells on the hybrid substrate of PVLA and EFC were examined by PCR (Figure 5A). It has been reported that the cellular transcription markers can be regulated in complex environments, including ones that contain hormones, cytokines, xenobiotics, and ECMs. We found that the cells on the coimmobilized matrix of PVLA and EFC maintained expression of liver-specific genes, including those for albumin, HNF-4 $\alpha$, and ASGPR, for at least 5 days (Figure 5A). Among all the hybrid systems with various concentrations of serum, the coimmobilized matrix with $1 \%$ FBS exhibited an optimal performance in preserving hepatic 
A
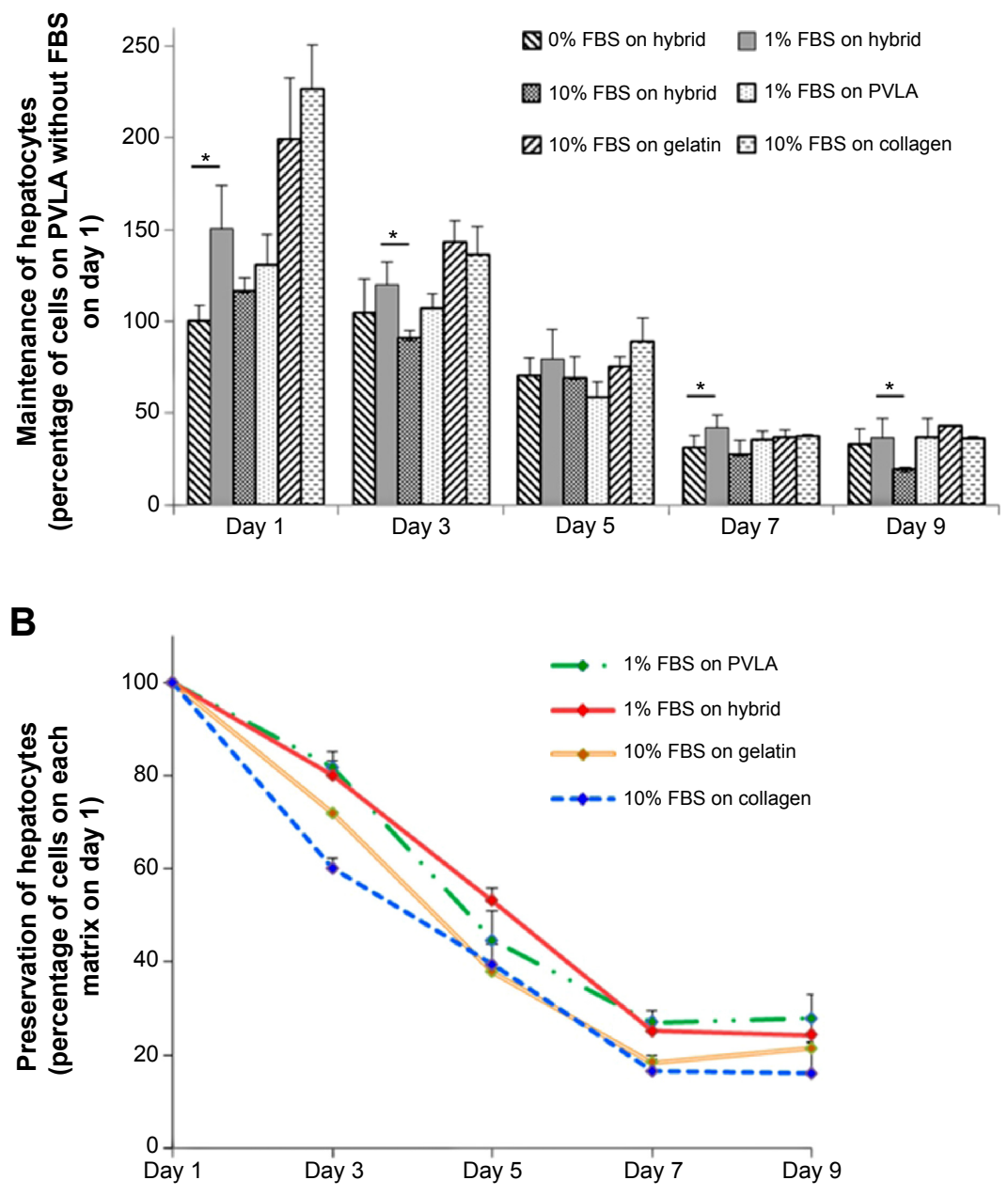

Figure 4 The maintenance of primary hepatocytes on the hybrid matrix after I, 3, 5, 7, and 9 days.

Notes: The relative ratios of attached cells in various systems were determined by using the number of cells on PVLA without FBS as the I00\% value. The number of primary hepatocytes on the hybrid matrix was higher in the medium with I\% FBS at every time point than the cells on the same surface without or with $10 \%$ FBS. The hepatocytes were viable on PVLA (I\% FBS), gelatin (I0\% FBS), and collagen (I0\% FBS), which was used as a control (A). The relative preservation of hepatocytes was determined by normalizing the cell numbers to the value after I day on each matrix (B). The hybrid matrix was made up of EFC and PVLA. The symbol $*$ denotes statistical significance at $P<0.05$.

Abbreviations: FBS, fetal bovine serum; PVLA, poly-(N-p-vinylbenzyl-4-O- $\beta$-D-galactopyranosyl-D-gluconamide); EFC, E-cadherin-Fc.

expression, especially after long-term incubation. Meanwhile, compared to the gelatin surface (10\% FBS), the PVLA/EFC matrix (1\% FBS) may have to a greater extent enhanced the expression levels of mature markers in primary hepatocytes, such as albumin (Figure 5B), HNF-4 $\alpha$ (Figure 5C), and ASGPR (Figure 5D) at each time point. During the incubation, the isolated hepatocytes on the hybrid substratum showed no sharp declines in the expression of these genes, whereas the expression rapidly decreased on the control surface. The preservation effect was particularly evident in the markers, including HNF-4 $\alpha$ (Figure 5C) and ASGPR (Figure 5D), that were expressed in highly mature hepatic cells.

To further examine the serum effect, the albumin expression in hepatocytes at the same time points (days 1 and 5) was revealed by the Western blot method (Figure 6) and was consistent with the immunochemistry results. On each matrix, albumin began to be secreted in abnormally high amounts after 1 day of incubation. The highest level of protein secretion was exhibited in cells maintained on the hybrid matrix supplied with $1 \%$ FBS, whereas the amount of secretion was attenuated in the hepatocytes with other serum concentrations or on other surfaces.

\section{The glycogen storage in hepatocytes on the hybrid matrix}

In addition to their protein secreting capability, hepatocytes possess many other functionalities, such as the synthesis of biomolecules, transformation of carbohydrates, and formation of bile. Here, the functional expression of primary hepatocytes was revealed by their glycogen storage capability after 
A RT-PCR results for hepatic markers

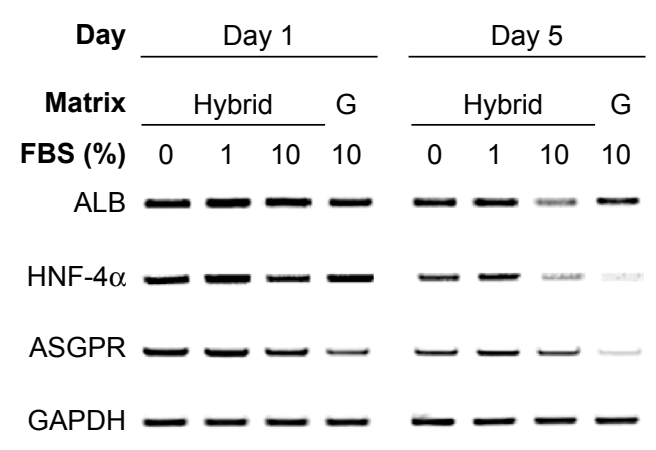

B

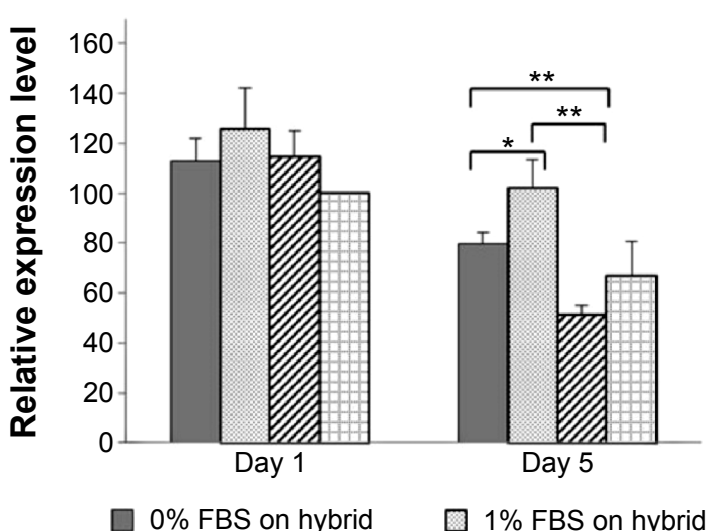

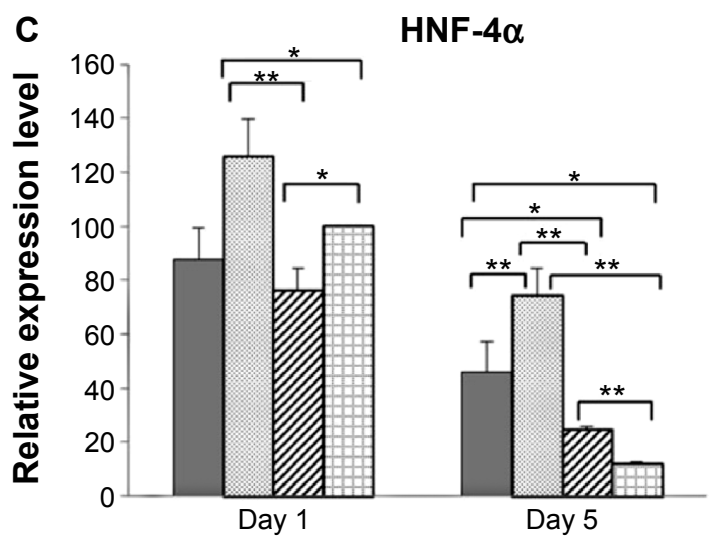

D ASGPR

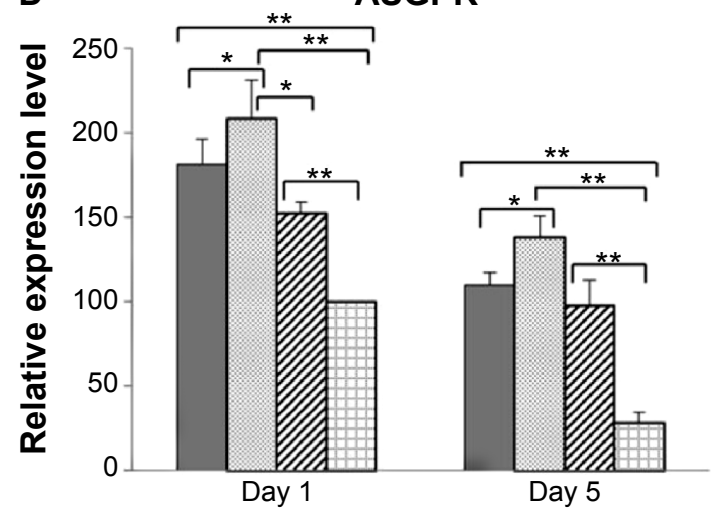

\10\% FBS on hybrid

⿴10\% FBS on $\mathrm{G}$

Figure 5 The transcription markers of primary hepatocytes on the hybrid matrix with different concentrations of FBS on days I and 5 .

Notes: The expression of hepatic markers at the gene level was analyzed by using RT-PCR (A). The relative expression levels of the markers ALB (B), HNF-4 $\alpha$ (C), and ASGPR (D) were quantified by using Imagej software and normalized to the GAPDH housekeeping gene. The primary hepatocytes cultured on gelatin with I0\% FBS are used as a control. The expression level of each maker on gelatin after I day culture is considered as I00\%. The hybrid matrix was made up of EFC and PVLA. The symbols $*$ and **denote statistical significance at $P<0.05$ and at $P<0.01$.

Abbreviations: RT-PCR, reverse transcription polymerase chain reaction; G, gelatin; FBS, fetal bovine serum; ALB, albumin; HNF-4 $\alpha$, hepatocyte nuclear factor-4 alpha; ASGPR, asialoglycoprotein receptor; GAPDH, glyceraldehyde 3-phosphate dehydrogenase; EFC, E-cadherin-Fc; PVLA, poly-(N-p-vinylbenzyl-4-O- 3 -D-galactopyranosyl-D-gluconamide).

1 day (Figure 7). The hepatocytes showed an elevated level of stored glycogen (red color) on the PVLA surface (Figure 7A) and on the coimmobilized matrix (Figure 7B) with a cluster morphology, in contrast to the spreading cells on the gelatin (Figure 7C) and on the collagen surface (Figure 7D). The amount of stored glycogen was highest in cells on the PVLA matrix, although the cell number there was the smallest.

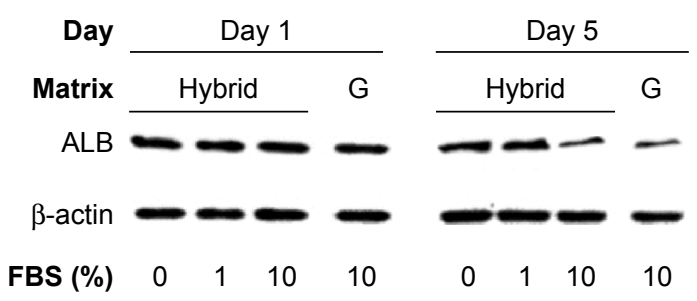

Figure 6 The ALB expression of hepatocytes after day I and after day 5. Notes: The ALB expression was confirmed using Western blot analysis and compared with the expression of the housekeeping $\beta$-actin.

Abbreviations: ALB, albumin; G, gelatin; FBS, fetal bovine serum.

\section{Discussion}

PVLA, which possesses galactose termini, plays an important role in hepatocyte incubation and stimulation because of its specific ability to recognize ASGPRs on the cellular membrane. ${ }^{24,25}$ The receptors may seize the galactose ends on the side chains of the glycopolymer and may tend to ingest them through cellular endocytosis. However, the hydrophobic interaction between the polymer backbone and PS can attach PVLA to the surface of nontreated cell culture dishes. Thus, the PVLA matrix possesses the capability to specifically adhere to the isolated hepatocytes and maintain the cellular expression of ASGPRs at a high level. The receptors commonly found on mature hepatocytes are believed to induce maturity of these cells. ${ }^{26}$ The PVLA matrix is hence suitable for the culture of primary hepatocytes and the maintenance of their hepatic differentiation status after isolation. ${ }^{27}$ Furthermore, a $\mathrm{Ca}^{2+}$ dependent cell 

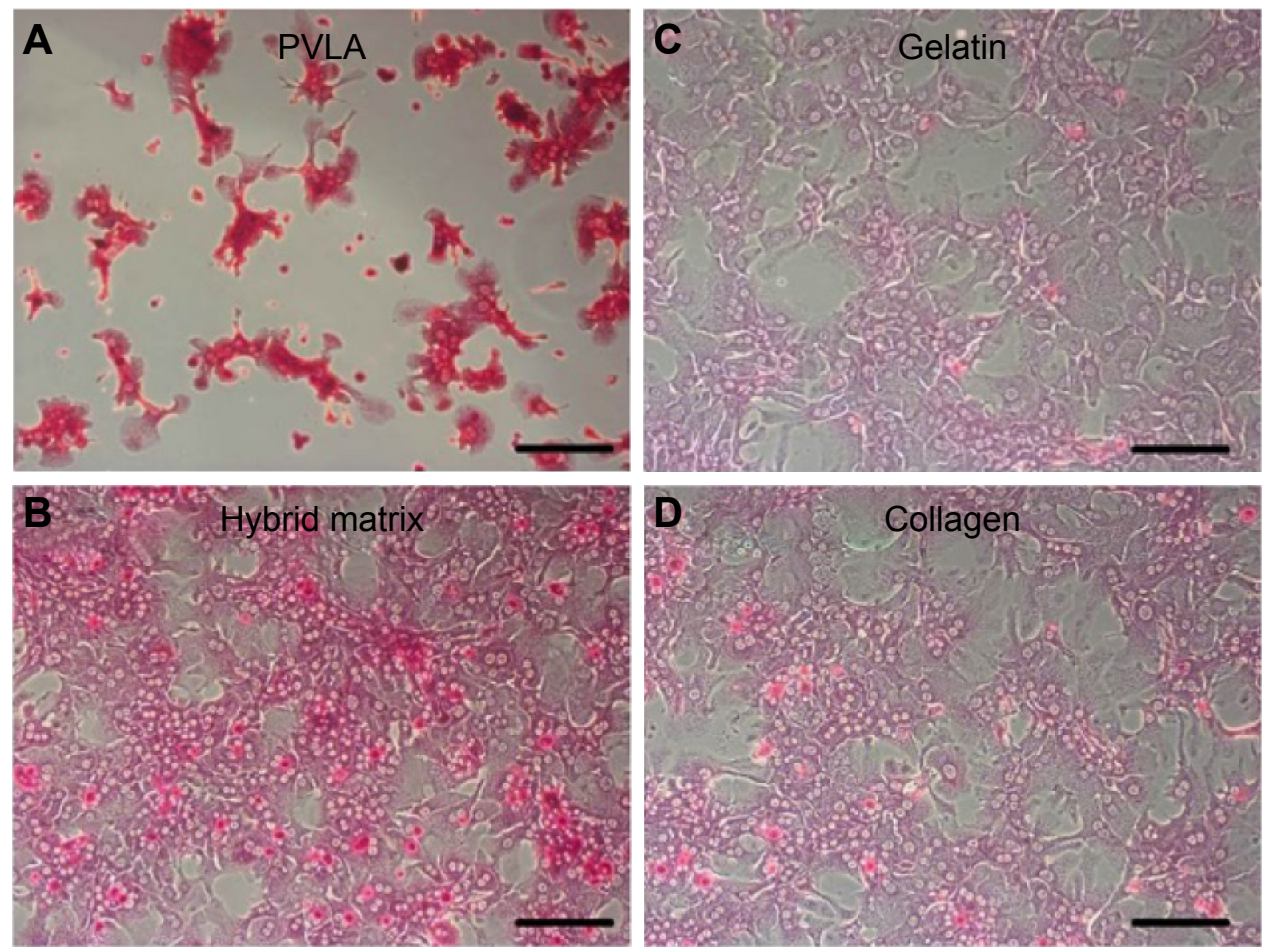

Figure 7 The storage of glycogen (red) in primary hepatocytes on various surfaces after I day in culture.

Notes: The surfaces include a PVLA-coated dish (A), the hybrid matrix (B), a gelatin-coated dish (C), and a collagen-coated dish (D). Scale bar: $50 \mu \mathrm{m}$. Abbreviation: PVLA, poly-(N-p-vinylbenzyl-4-O- $\beta$-D-galactopyranosyl-D-gluconamide).

adhesion molecule, E-cadherin, also contributes to the formation of aggregates in endoderm cell lineages and hence results in a prolongation of the cell survivability term and further enhancement of differentiation functionality. ${ }^{14,28} \mathrm{~A}$ chimeric protein called EFC was developed with E-cadherin as a base by combining the extracellular domain of mouse E-cadherin and the Fc domain of IgG. The fusion protein was intended to be applied for cell recognition and incubation. The artificial substratum may selectively recognize endoderm cells and further determine the differentiation direction of stem cells in the endodermal system, including cells of hepatic lineage. ${ }^{12}$ Therefore, we combined the virtues of E-cadherin and galactose-carrying biomaterials in the hepatic culture. Specifically, the hybrid system for mouse primary hepatocytes was devised by immobilizing EFC molecules after PVLA addition onto a hydrophobic surface, and culture media without any cytokines but various concentrations (v/v $0 \%, 1 \%$, and $10 \%)$ of FBS were used. The beneficial effects of the coimmobilized substratum on the cellular phenotype, serum dependency, and functional expression were revealed at both the gene and protein levels.

First, the attachment of hepatocytes is significant in primary culture because further survival of the cells is dependent on the initial attachment status. It is believed that cellular adhesion is mediated through the specific recognition of ECM molecules by receptors on the cellular membrane. The attachment of cells onto natural substrata depends on the competitive adsorption of proteins (including the proteins that constitute the ECMs) that are added to the culture medium or secreted by cells. The bioactive compounds in serum have been confirmed to significantly affect the cellular behaviors on ECMs such as collagen, gelatin, and fibronectin. In addition to the action of cellular attachment, FBS also provides essential nutrients for cell growth. Therefore, increasing the serum amount within the environment may enhance the number of attached cells, and a concentration of $10 \%$ FBS may induce most of the viable cells to anchor to the traditional matrices.

Second, our results regarding hepatocytes on the hybrid matrix and PVLA revealed a reduced need for serum to produce cell attachment (Figure 2). Consistent with nature, hepatocyte attachment on PVLA is not mediated by integrin, and anoikis is further suppressed in the system. ${ }^{17,29}$ It has been reported that only the hepatocytes with a high maturity level can steadily attach to PVLA surfaces because of the large number of ASGPRs present on the cellular membrane. The selective effect of the polymer is the reason that cell attachment to the 
pure PVLA matrix is limited. Thus, the number of attached cells was less than the number of isolated liver cells, which consist of hepatocytes of both high and low maturity levels and nonparenchymal cells. ${ }^{30}$ The addition of E-cadherin to the system could attract more hepatocytes with an inadequate amount of ASGPRs to attach to the matrix because hepatocytes belong to the endoderm cell lineage. Thus, the limitation on cellular attachment was extended; however, among the hybrid systems with different FBS concentration, the largest amount of cell attachment was found on the matrix supplied with 1\% FBS; this amount was still lower than the number of cells attached to the gelatin and collagen surfaces with $10 \%$ serum.

Subsequently, the cell number decreased after the incubation period because the isolated mouse hepatocytes lost their proliferating capability in vitro unless specific factors are added, such as spleen derived growth factor (but not the serum), ${ }^{31}$ the transgenes of c-myc and transforming growth factor- $\alpha,{ }^{32}$ or coculture with feeder cells. ${ }^{33}$ It has been reported that the addition of other bioactive molecules, such as epidermal growth factor, insulin, or serum, may delay cell apoptosis and promote the cell's functional expression. However, the application of serum can possibly introduce inhomogeneity and immunogenicity and raise the cost of the culture system. Therefore, it remains important to reduce the addition of serum during the incubation of primary hepatocytes after attachment. In this study, the PVLA surface could tightly immobilize the isolated hepatocytes after cell capture. The cadherin-mediated cellular adhesion on the coimmobilized matrix had the capability to enhance cell maintenance in the culture system without causing cell detachment and resulted in the morphological differences among the galactose-carrying matrices. Finally, after 1 week in culture or longer, the number of residual hepatocytes declined to a similar cellular density with scarcely any hepatic markers on each combination of substratum and FBS concentration (Figure 4). Therefore, the hybrid substratum exhibited an excellent capacity to maintain the cell number and low serum dependency as well.

In the prolonged period of culture, the cells spread on gelatin-coated dishes gradually lost their abilities for hepatic expression at both the protein (Figure 3) and gene levels (Figure 5), whereas the hybrid system and PVLA matrix significantly promoted liver functionality. The preservation period of marker expression also supported the role of PVLA in forming aggregates and maintaining the hepatic functionality of cells. Meanwhile, the E-cadherin in the matrix helped to maintain the cells in a monolayer morphology, which kept the cells in a homogeneous environment. Therefore, it was demonstrated that the cellular interactions inside the aggregates in both multilayers and monolayers, which were promoted by PVLA and EFC, may bestow a remarkable regulating effect on the marker expression of hepatocytes. $^{34,35}$ Finally, the interaction between galactose and ASGPRs possibly ensured that hepatocytes with a high maturity level remained on the dish surface and functional expression was highly preserved (Figure 7).

We also demonstrated that $1 \%$ may be the optimal FBS concentration to preserve protein secretion in hepatocyte culture on a hybrid matrix; the results from this combination were better than those for the general matrix consisting of a gelatin-coated culture dish (Figure 6). The highest expression level observed in the hybrid system with a small quantity of FBS in the medium was speculated to be the result of the initiating and nourishing effect of serum in a very low concentration. The ability of the hybrid system to maintain the hepatocytes under low-serum or serum-free conditions allows the elimination of the negative effects of animal products in clinical applications and establishment of a completely defined culture system for hepatocytes.

\section{Conclusion}

The coimmobilized matrix combined the advantages of PVLA for maintaining hepatic function and of EFC for enhancing cellular viability with a low requirement for FBS in vitro. For mouse primary hepatocytes, it provided the ideal culture conditions, which are able to support cell proliferation and differentiation and further prevent interference and infection of biological products. The hybrid system may be further applied in the induction of stem cells to hepatocytes and has the advantages of promoting the affinity of hepatic cells and allowing low dependency on serum.

\section{Acknowledgments}

This work was supported by Grants-in-aid for Scientific Research from the Ministry of Education, Culture, Sports, Science and Technology of Japan, Tsinghua University Initiative Scientific Research Program (20121087982), the National Basic Research Program of China (973 Program, 2011CB606205) and China Postdoctoral Science Foundation (2013M540090).

\section{Disclosure}

The authors report no conflicts of interest in this work.

\section{References}

1. Ourlin JC, Vilarem MJ, Daujat M, et al. Lipid-mediated transfection of normal adult human hepatocytes in primary culture. Anal Biochem. 1997;247(1):34-44.

2. Sasaki K, Kitaguchi Y, Fukuda T, Aoyagi Y. Ascorbic acid supplementation to primary culture of chicken hepatocytes with non-serum medium. Int J Biochem Cell Biol. 2000;32(9):967-973. 
3. Hou DX, Kunitake T, Kusuda J, Fujii M. Primary culture of chicken hepatocytes as an in vitro model for determining the influence of dioxin. Biosci Biotechnol Biochem. 2001;65(1):218-221.

4. Tokiwa T, Kano J, Meng XY, Kodama M. Primary culture of adult rat hepatocytes on porous expanded polytetrafluoroethylene. Transplant Proc. 1997;29(4):2120-2122.

5. Shang Y, Tamai M, Ishii R, et al. Hybrid sponge comprised of galactosylated chitosan and hyaluronic acid mediates the co-culture of hepatocytes and endothelial cells. J Biosci Bioeng. 2014;117(1):99-106.

6. Kim SH, Hoshiba T, Akaike T. Hepatocyte behavior on synthetic glycopolymer matrix: inhibitory effect of receptor-ligand binding on hepatocyte spreading. Biomaterials. 2004;25(10):1813-1823.

7. Yang W, Mou T, Shao G, Wang F, Zhang X, Liu B. Copolymer-based hepatocyte asialoglycoprotein receptor targeting agent for SPECT. J Nucl Med. 2011;52(6):978-985.

8. $\mathrm{Mu} \mathrm{H}$, Lin KX, Zhao H, et al. Identification of biomarkers for hepatocellular carcinoma by semiquantitative immunocytochemistry. World J Gastroenterol. 2014;20(19):5826-5638.

9. Windler E, Greeve J, Levkau B, Kolb-Bachofen V, Daerr W, Greten H. The human asialoglycoprotein receptor is a possible binding site for low-density lipoproteins and chylomicron remnants. Biochem J. 1991; 276(Pt 1):79-87.

10. Kim SH, Kim JH, Akaike T. Regulation of cell adhesion signaling by synthetic glycopolymer matrix in primary cultured hepatocyte. FEBS Lett. 2003;553:433-449.

11. Mizumoto H, Hayashi S, Matsumoto K, et al. Evaluation of a hybrid artificial liver module based on a spheroid culture system of embryonic stem cell-derived hepatic cells. Cell Transplant. 2012;21(2-3):421-428.

12. Haque A, Hexig B, Meng Q, Hossain S, Nagaoka M, Akaike T. The effect of recombinant E-cadherin substratum on the differentiation of endoderm-derived hepatocyte-like cells from embryonic stem cells. Biomaterials. 2011;32(8):2032-2042.

13. Semler EJ, Dasgupta A, Moghe PV. Cytomimetic engineering of hepatocyte morphogenesis and function by substrate-based presentation of acellular E-cadherin. Tissue Eng. 2005;11(5-6):734-750.

14. Meng Q, Haque A, Hexig B, Akaike T. The differentiation and isolation of mouse embryonic stem cells toward hepatocytes using galactosecarrying substrata. Biomaterials. 2012;33(5):1414-1427.

15. Kohno Y, Shiraki K, Mura T. The effect of human milk on DNA synthesis of neonatal rat hepatocytes in primary culture. Pediatr Res. 1991;29(3):251-255.

16. Brocks DR, Hamdy DA, Ben-Eltriki M, Patel JP, El-Kadi AO. Effect of rat serum lipoproteins on mRNA levels and amiodarone metabolism by cultured primary rat hepatocytes. J Pharm Sci. 2013;102(1):262-270.

17. Hoshiba T, Nagahara H, Cho CS, Tagawa Y, Akaike T. Primary hepatocyte survival on non-integrin-recognizable matrices without the activation of Akt signaling. Biomaterials. 2007;28(6):1093-1104.

18. Hirose S, Ise H, Uchiyama M, Cho CS, Akaike T. Regulation of asialoglycoprotein receptor expression in the proliferative state of hepatocytes. Biochem Biophys Res Commun. 2001;287(3):675-681.

19. Kobayashi A, Kobayashi K, Akaike T. Control of adhesion and detachment of parenchymal liver cells using lactose-carrying polystyrene as substratum. J Biomater Sci Polym Ed. 1992;3(6):499-508.
20. Nagaoka M, Akaike T. Single amino acid substitution in the mouse IgG1 Fc region induces drastic enhancement of the affinity to protein A. Protein Eng. 2003;16(4):243-245.

21. Seglen PO. Preparation of rat liver cells. 3. Enzymatic requirements for tissue dispersion. Exp Cell Res. 1973;82(2):391-398.

22. Shen L, Hillebrand A, Wang DQ, Liu M. Isolation and primary culture of rat hepatic cells. J Vis Exp. 2012;64:84-88.

23. Klaunig JE, Goldblatt PJ, Hinton DE, Lipsky MM, Trump BF. Mouse liver cell culture. II. Primary culture. In Vitro. 1981;17(10):926-934.

24. Nagaki M, Shidoji Y, Yamada Y, et al. Regulation of hepatic genes and liver transcription factors in rat hepatocytes by extracellular matrix. Biochem Biophys Res Commun. 1995;210(1):38-43.

25. Lee JS, Kim SH, Kim YJ, Akaike T, Kim SC. Hepatocyte adhesion on a poly[N-p-vinylbenzyl-4-O-beta-D-galactopyranosyl-D-glucoamide]coated poly(L-lactic acid) surface. Biomacromolecules. 2005;6(4): 1906-1911.

26. Bartles JR, Zhang LQ, Verheyen EM, Hospodar KS, Nehme CL, Fayos BE. Decreases in the relative concentrations of specific hepatocyte plasma membrane proteins during liver regeneration: downregulation or dilution? Dev Biol. 1991;143(2):258-270.

27. Ise H, Nikaido T, Negishi N, et al. Effective hepatocyte transplantation using rat hepatocytes with low asialoglycoprotein receptor expression. Am J Pathol. 2004;165(2):501-510.

28. Takei R, Suzuki D, Hoshiba T, et al. Role of E-cadherin molecules in spheroid formation of hepatocytes adhered on galactose-carrying polymer as an artificial asialoglycoprotein model. Biotechnol Lett. 2005; 27(16):1149-1156.

29. Leung-Hagesteijn CY, Milankov K, Michalak M, Wilkins J, Dedhar S. Cell attachment to extracellular matrix substrates is inhibited upon downregulation of expression of calreticulin, an intracellular integrin alpha-subunit-binding protein. J Cell Sci. 1994;107(Pt 3):589-600.

30. Kim SJ, Ise H, Goto M, Akaike T. Interactions of vimentin- or desminexpressing liver cells with $\mathrm{N}$-acetylglucosamine-bearing polymers. Biomaterials. 2012;33(7):2154-2164.

31. Suzuki T, Koga N, Imamura T, Mitsui Y. A novel growth factor in rat spleen which promotes proliferation of hepatocytes in primary culture. Biochem Biophys Res Commun. 1988;153(3):1123-1128.

32. Kao CY, Factor VM, Thorgeirsson SS. Reduced growth capacity of hepatocytes from c-myc and c-myc/TGF-alpha transgenic mice in primary culture. Biochem Biophys Res Commun. 1996;222:64-70.

33. Takagi M, Kondo H, Yoshida T. In vitro proliferation of primary rat hepatocytes expressing ureogenesis activity by coculture with STO cells. J Biosci Bioeng. 2002;94(3):212-217.

34. Ise H, Sugihara N, Negishi N, Nikaido T, Akaike T. Low asialoglycoprotein receptor expression as markers for highly proliferative potential hepatocytes. Biochem Biophys Res Commun. 2001;285(2):172-182.

35. Luebke-Wheeler JL, Nedredal G, Yee L, Amiot BP, Nyberg SL. E-cadherin protects primary hepatocyte spheroids from cell death by a caspase-independent mechanism. Cell Transplant. 2009;18(12): 1281-1287.
International Journal of Nanomedicine

\section{Publish your work in this journal}

The International Journal of Nanomedicine is an international, peerreviewed journal focusing on the application of nanotechnology in diagnostics, therapeutics, and drug delivery systems throughout the biomedical field. This journal is indexed on PubMed Central, MedLine, CAS, SciSearch ${ }^{\circledR}$, Current Contents ${ } /$ Clinical Medicine,

\section{Dovepress}

Journal Citation Reports/Science Edition, EMBase, Scopus and the Elsevier Bibliographic databases. The manuscript management system is completely online and includes a very quick and fair peer-review system, which is all easy to use. Visit http://www.dovepress.com/ testimonials.php to read real quotes from published authors. 